\title{
Observation of Fermi Polarons in a Tunable Fermi Liquid of Ultracold Atoms
}

\author{
André Schirotzek, Cheng-Hsun Wu, Ariel Sommer, and Martin W. Zwierlein \\ Department of Physics, MIT-Harvard Center for Ultracold Atoms, and Research Laboratory of Electronics, \\ Massachusetts Institute of Technology, Cambridge, Massachusetts 02139, USA
}

(Received 17 February 2009; revised manuscript received 9 April 2009; published 8 June 2009)

\begin{abstract}
We have observed Fermi polarons, dressed spin-down impurities in a spin-up Fermi sea of ultracold atoms. The polaron manifests itself as a narrow peak in the impurities' rf spectrum that emerges from a broad incoherent background. We determine the polaron energy and the quasiparticle residue for various interaction strengths around a Feshbach resonance. At a critical interaction, we observe the transition from polaronic to molecular binding. Here, the imbalanced Fermi liquid undergoes a phase transition into a Bose liquid, coexisting with a Fermi sea.
\end{abstract}

DOI: 10.1103/PhysRevLett.102.230402

The fate of a single impurity interacting with its environment determines the low-temperature behavior of many condensed matter systems. A well-known example is given by an electron moving in a crystal lattice, displacing nearby ions and thus creating a localized polarization. The electron, together with its surrounding cloud of lattice distortions, phonons, forms the lattice polaron [1]. It is a quasiparticle with an energy and mass that differ from that of the bare electron. Polarons are central to the understanding of colossal magnetoresistance materials [2], and they affect the spectral function of cuprates, the parent material of high- $T_{C}$ superconductors [3]. Another famous impurity problem is the Kondo effect, where immobile spin impurities give rise to an enhanced resistance in metals below the Kondo temperature [4]. In contrast to the electron moving in a phonon bath, a bosonic environment, in the latter case the impurity interacts with a fermionic environment, the Fermi sea of electrons.

Here we study a small concentration of spin-down impurities immersed in a spin-up Fermi sea of ultracold atoms. This system represents the limiting case of spinimbalanced Fermi gases and has been recognized to hold the key to the quantitative understanding of the phase diagram of imbalanced Fermi mixtures [5-16]. Unlike in liquid ${ }^{3} \mathrm{He}$, the $s$-wave interaction potential between the impurities and the spin-up atoms in this novel spinimbalanced Fermi liquid is attractive. The vicinity of a Feshbach resonance allows tuning of the interaction strength at will, characterized by the ratio of the interparticle distance $\sim 1 / k_{F}$ to the scattering length $a$, where $k_{F}$ is the spin-up Fermi wave vector [17]. Figure 1 depicts the scenario for a single impurity: For weak attraction $\left(1 / k_{F} a \ll-1\right)$ the impurity propagates freely in the spin-up medium of density $n_{\uparrow}=k_{F}^{3} / 6 \pi^{2}$ [Fig. 1(a)]. It merely experiences the familiar attractive mean field energy shift $E_{\downarrow}=4 \pi \hbar^{2} a n_{\uparrow} / m<0$. However, as the attractive interaction grows, the impurity can undergo momentum changing collisions with environment atoms, and thus starts to attract its surroundings. The impurity "dressed" with the localized cloud of scattered fermions constitutes
PACS numbers: 05.30.Fk, 03.75.Ss, 32.30.Bv, 67.60.Fp

the Fermi polaron [Fig. 1(b)]. Dressing becomes important once the mean free path $\sim 1 / n_{\uparrow} a^{2}$ of the bare impurity in the medium becomes comparable to the distance $\sim 1 / k_{F}$ between environment particles or when $\left(k_{F} a\right)^{2} \sim 1$. Collisions then reduce the bare impurity's probability of free propagation, the quasiparticle residue $Z$, from unity. The dressed impurity can instead move freely through the environment, with an energy $E_{\downarrow}$ shifted away from the simple mean field result. This polaronic state is stable until, for strong attraction $\left(1 / k_{F} a \sim 1\right)$, equivalent to a deep effective potential well, the spin-down impurity will bind exactly one spin-up atom, thus forming a tightly bound molecule [Fig. 1(c)]. This molecule is itself a dressed impurity, albeit a bosonic one [13].

To prepare and observe Fermi polarons, we start with a spin-polarized cloud of ${ }^{6} \mathrm{Li}$ atoms in the lowest hyperfine state $|1\rangle$ (spin-up), confined in a cylindrically symmetric optical trap $(125 \mu \mathrm{m}$ waist, $145 \mathrm{~Hz} / 22.3 \mathrm{~Hz}$ radial/axial trapping frequency) at a magnetic field of $690 \mathrm{G}$ [17]. A two-photon Landau-Zener sweep transfers a small fraction into state $|3\rangle$ (spin-down), and further cooling results in a cloud containing $2 \%|3\rangle$ impurities immersed in a degenerate Fermi gas of $5 \times 10^{6}|1\rangle$ atoms at a temperature $T=$ $0.14(3) T_{F}$, where $T_{F}$ is the Fermi temperature. A $100 \mathrm{G}$ wide Feshbach resonance for scattering between these states is centered at $690 \mathrm{G}$. For various fields around the

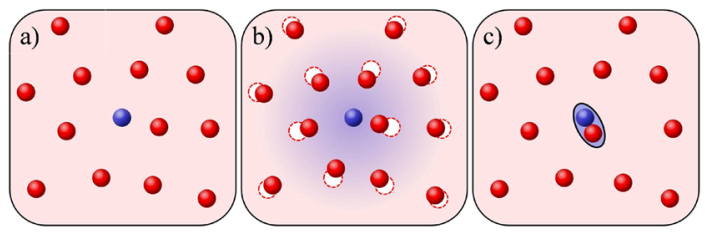

FIG. 1 (color online). From polarons to molecules. (a) For weak attraction, an impurity (blue) experiences the mean field of the medium (red). (b) For stronger attraction, the impurity surrounds itself with a localized cloud of environment atoms, forming a polaron. (c) For strong attraction, molecules of size $a$ form despite Pauli blocking of momenta $\hbar k<\hbar k_{F} \ll \hbar / a$ by the environment. 
resonance, we perform rf spectroscopy on the impurity species $|3\rangle$ and on the environment particles in $|1\rangle$ by transferring atoms into the empty state $|2\rangle$, accessible to either hyperfine state. This state is sufficiently weakly interacting with the initial states to allow a direct interpretation of the resulting spectra [18]. As in previous work, spectra are spatially resolved and tomographically 3D reconstructed [19] via an inverse Abel transform, and are thus local and free from broadening due to density inhomogeneities. In addition, phase contrast images yield the in situ density distribution $n_{\uparrow}, n_{\downarrow}$ and thus the local Fermi energy $\epsilon_{F}$ of the environment atoms and the local impurity concentration $x=\frac{n_{\downarrow}}{n_{1}}$. The Rabi frequencies $\Omega_{R}$ for the impurity and environment $\mathrm{rf}$ transitions are measured (on fully polarized samples) to be identical to within $5 \%$.

Figure 2 shows the observed spectra of the spin-down impurities and that of the spin-up environment at low local impurity concentration. The bulk of the environment spectrum is found at zero offset, corresponding to the free (Zeeman plus hyperfine) energy splitting between states $|1\rangle$ and $|2\rangle$. However, interactions between impurity and spin-up particles lead to a spectral contribution that is shifted: The rf photon must supply additional energy to transfer a particle out of its attractive environment into the final, noninteracting state [17]. In Fig. 2(a), impurity and environment spectra above zero offset exactly overlap, signaling two-body molecular pairing. The steep threshold gives the binding energy, the high-frequency wings arise from molecule dissociation into remnants with nonzero momentum $[17,20,21]$. As the attractive interaction is reduced, however, a narrow peak appears in the impurity spectrum that is not matched by the response of the environment [Figs. 2(b)-2(d)]. This narrow peak, emerging from a broad incoherent background, signals the formation of the Fermi polaron, a long-lived quasiparticle. The narrow width and long lifetime are expected: At zero temperature the zero momentum polaron has no phase space for decay and is stable. At finite kinetic energy or finite temperature $T$ it may decay into particle-hole excitations [13], but phase space restrictions due to the spin-up Fermi sea and conservation laws imply a decay rate $\propto\left(T / T_{F}\right)^{2} \sim$
$1 \%$ in units of the Fermi energy. Indeed, the width of the polaron peak is consistent with a delta function within the experimental resolution, as calibrated by the spectra of fully polarized clouds. The background is perfectly matched by the rf spectrum of the environment. This is expected at high rf energies $\hbar \omega \gg \epsilon_{F}$ that are probing high momenta $k \gg k_{F}$ and thus distances short compared to the interparticle spacing. Here, an impurity particle will interact with only one environment particle, leading to overlapping spectra.

Chevy has provided an instructive variational wave function $[5,9]$ that captures the essential properties of the polaron, even on a quantitative level [16] when compared with Monte Carlo (MC) calculations [6,12,13]:

$$
|\Psi\rangle=\varphi_{0}|\mathbf{0}\rangle_{\downarrow}|F S\rangle_{\uparrow}+\sum_{|\mathbf{q}|<k_{F}<|\mathbf{k}|} \varphi_{\mathbf{k q}} c_{\mathbf{k} \uparrow}^{\dagger} c_{\mathbf{q} \uparrow}|\mathbf{q}-\mathbf{k}\rangle_{\downarrow}|F S\rangle_{\uparrow}
$$

The first part describes a single impurity with a welldefined wave vector $\left(\mathbf{k}_{\downarrow}=\mathbf{0}\right)$ that is not localized and free to propagate in the Fermi sea of up spins $|F S\rangle_{\uparrow}$. In the second part the impurity particle recoils off environment particles that are scattered out of the Fermi sea and leave holes behind. This describes the dressing of the impurity with particle-hole excitations. The probability of free propagation is given by the first, unperturbed part, $Z=$ $\left|\varphi_{0}\right|^{2}$. According to Fermi's golden rule $[11,15,17,22]$, the two portions of $|\Psi\rangle$ give rise to two distinct features of the impurity rf spectrum $\Gamma(\omega)$ ( $\omega$ is the rf offset from the bare atomic transition):

$$
\Gamma(\omega)=2 \pi \hbar \Omega_{R}^{2} Z \delta\left(\hbar \omega+E_{\downarrow}\right)+\Gamma^{\mathrm{inc}}(\omega) .
$$

The first part in $|\Psi\rangle$ contributes a coherent narrow quasiparticle peak to the minority spectrum. Its position is a direct measure of the polaron energy $E_{\downarrow}$, its integral gives the quasiparticle residue $Z$. The particle-hole excitations in the second part give rise to a broad, incoherent background $\Gamma^{\text {inc }}(\omega) \propto \sum_{\mathbf{q}, \mathbf{k}}\left|\varphi_{\mathbf{q k}}\right|^{2} \delta\left(\hbar \omega-\epsilon_{\mathbf{q}-\mathbf{k}}-\epsilon_{\mathbf{k}}+\epsilon_{\mathbf{q}}+E_{\downarrow}\right)$ : The polaron energy $E_{\downarrow}$ is released as the impurity at momentum $\mathbf{q}-\mathbf{k}$ is transferred into the final state, leaving behind an
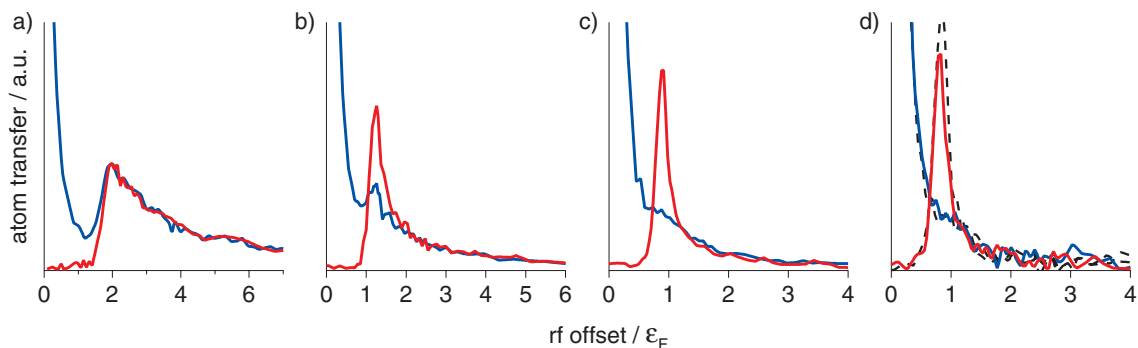

FIG. 2 (color online). rf spectroscopy on polarons. Shown are spatially resolved, 3D reconstructed rf spectra of the environment (blue, state $|1\rangle$ ) and impurity (red, state $|3\rangle$ ) component in a highly imbalanced spin-mixture. (a) Molecular limit; (b), (c) Emergence of the polaron, a distinct peak exclusively in the minority component. (d) At unitarity, the polaron peak is the dominant feature in the impurity spectrum, which becomes even more pronounced for $1 / k_{F} a<0$ (not shown). For the spectra shown as dashed lines in (d) the roles of states $|1\rangle$ and $|3\rangle$ are exchanged. The local impurity concentration was $x=5(2) \%$ for all spectra, the interaction strengths $1 / k_{F} a$ were (a) $0.76(2)$, (b) $0.43(1)$, (c) $0.20(1)$, and (d) 0 (unitarity). 
environment particle in $\mathbf{k}$ above and a hole within the Fermi sea at $\mathbf{q}$ [22]. These two spectral features are recovered in theoretical rf spectra for a finite number of impurities, i.e., a Fermi liquid [15,23]. For our analysis we do not rely on a theoretical fit to the spectra.

To measure the polaron energy $E_{\downarrow}$, we determine the peak position of the impurity spectrum as a function of the local interaction parameter $1 / k_{F} a$. The data for $5 \% \mathrm{impu-}$ rity concentration are shown in Fig. 3(a), along with the variational upper bound given by the wave function Eq. (1) [22] and the diagrammatic MC calculation of [13]. As final state interactions are weak, they can be included as a simple repulsive mean field shift $4 \pi \hbar^{2} a_{\mathrm{fe}} n_{\uparrow} / m$, with $a_{\mathrm{fe}}$ the scattering length between the final state and the environment atoms [24]. Polaron energies have been predicted via the variational ansatz [5], the $T$-matrix approach $[9,10,15,16]$, the $1 / N$ expansion [11], fixed-node MC $[6,12]$ and diagrammatic MC calculations [13]. With the exception of the $1 / N$ expansion, these all agree with each other and with the present experiment to within a few percent. In particular, in the unitary limit where $1 / k_{F} a=0$ we find a polaron energy of $E_{\downarrow}=-0.64(7) \epsilon_{F}\left(-0.72(9) \epsilon_{F}\right)$ when state $|3\rangle(|1\rangle)$ serves as the impurity [25]. This agrees well with the diagrammatic MC calculation, $-0.615 \epsilon_{F}$ [14], and the analytical result $-0.6156(2) \epsilon_{F}[16]$. Analy-

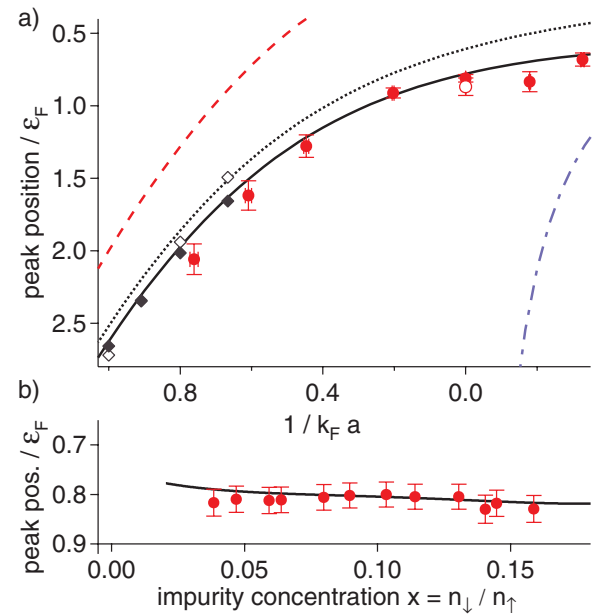

FIG. 3 (color online). Peak position of the impurity spectrum as a measure of the polaron energy $E_{\downarrow}$. (a) peak position for various interaction strengths in the limit of low concentration $x=5(2) \%$ (solid circles). Open circle: Reversed roles of impurity and environment. Dotted line: polaron energy from variational ansatz Eq. (1) [5], the solid line including weak final state interactions. Dashed line: Energy of a bare, isolated molecule in vacuum. Blue dash-dotted line: Mean field limit for the energy of an impurity atom. Solid (open) diamonds: Diagrammatic MC energy of the polaron (molecule) [13]. (b) Peak position at unitarity $\left(1 / k_{F} a=0\right)$ as a function of impurity concentration (solid circles). The line shows the expected peak position, $\hbar \omega_{p} / \epsilon_{F}=A+\left(1-\frac{m}{m^{*}}\right) x^{2 / 3}-\frac{6}{5} F x+\frac{4}{3 \pi} k_{F} a_{\mathrm{fe}}$, using the MC value $A=0.615$ [13], the analytic result $m^{*}=1.2$ [16], the weak repulsion between polarons with $F=0.14$ [12] and weak final state interactions with scattering length $a_{\mathrm{fe}}$. sis of experimental density profiles yields a value of $-0.58(5) \epsilon_{F}[26]$.

The relatively large value for $E_{\downarrow}$ directly implies that the normal state, modeled as a Fermi sea of weakly interacting polarons, is favored over the superfluid state up to a critical concentration (44\%), much higher than that predicted by mean field theories (4\%) [27]. These neglect interactions in the normal state and therefore imply a polaron binding energy of zero.

We have so far considered the limit of few impurities. By increasing their density, we can study the effect of interactions between polarons. In Fig. 3(b) we show that the quasiparticle peak position depends only weakly on the impurity concentration in the unitarity limit. Polarons are thus weakly interacting quasiparticles, despite the strong interactions between the bare impurity and its environment.

The peak position could be modified due to the effective mass $m^{*}$ of polarons, larger than the mass of the bare impurity. Transfer of a moving polaron into the free final state then requires additional kinetic energy. This leads to an upshift and a broadening on the order of the Fermi energy difference between initial and final state, $x^{2 / 3} \epsilon_{F}\left(1-\frac{m}{m^{*}}\right)$. On resonance, this is $0.04 \epsilon_{F}$ for $x=0.1$. The effect could be partially masked by the predicted weak repulsion between polarons [12] that would downshift the resonance frequency by $-0.02 \epsilon_{F}$ for $x=0.1$.

The spectral weight of the polaron peak directly gives the quasiparticle residue $Z$, a defining parameter of a Fermi liquid. Experimentally, we determine the area under the impurity peak that is not matched by the environment's

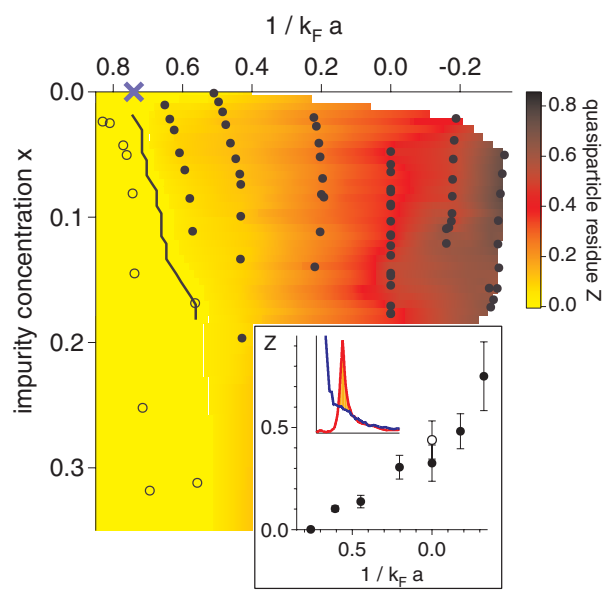

FIG. 4 (color online). Quasiparticle residue $Z$ as a function of interaction strength and impurity concentration. The color coding indicates the magnitude of $Z$ and is an interpolation of the data points shown in the graph. Open circles: Data points consistent with zero $(Z<0.03)$, solid circles: $Z>0.03$, the solid line marking the onset of $Z$. Blue cross: Critical interaction strength for the Fermi liquid-molecular BEC transition for $x \rightarrow$ 0 [12]. Inset: $Z$ as a function of interaction strength in the limit of low impurity concentration $x=5(2) \%$. Open circle: Reversed roles, $|1\rangle$ impurity in $|3\rangle$ environment. The spectrum in the inset illustrates the determination of $Z$ [22]. 
response and divide by the total area under the impurity spectrum (see spectrum in Fig. 4 and [22]). Figure 4 presents $Z$ as a function of interaction strength and impurity concentration $x$, the inset shows $Z$ for $x=5 \%$. As expected, $Z$ approaches $100 \%$ for weak attractive interaction $k_{F} a \rightarrow 0^{-}$, where the bare impurity only rarely recoils off environment atoms. As the mean free path shortens and the bare impurity starts to surround itself with environment atoms $Z$ decreases. On resonance, we find $Z=0.39(9)$ for $x=5 \%$, with only a weak dependence on $x$ (Fig. 4). Theoretical values for $Z$ vary: ansatz Eq. (1) predicts $Z=$ 0.78 for a single impurity, while Ref. [11] predicts $Z=$ $0.47(0.30)$ for vanishing $(5 \%)$ impurity concentration. Our procedure might yield a lower bound on the actual value of $Z$, as the incoherent part of the impurity spectrum might be depleted around threshold. Eventually, for strong attraction between the impurity and particles of the medium, $Z$ vanishes and we observe complete overlap of the impurity and environment spectra. This signals the formation of a two-body bound state between the impurity and exactly one environment atom. For a spin-down concentration of $5 \%$ we determine the critical interaction strength where the polaron peak vanishes to be $1 /\left(k_{F} a\right)_{c}=0.76(2)$. This is in good agreement with the independently determined critical interaction $1 / k_{F} a=0.74(4)$ beyond which one finds a superfluid even for the smallest impurity concentration [28]. This is a multicritical point $[12,13,29]$ where a Fermi liquid of weakly interacting polarons undergoes a phase transition into a Bose liquid of molecular impurities. Fixed-node $\mathrm{MC}$ calculations place this transition at a value of $1 / k_{F} a=0.73$ for $x \rightarrow 0$ [12]. Our $1 /\left(k_{F} a\right)_{c}$ is lower than the value 0.90 (2) from diagrammatic MC [13] for a single impurity. Ansatz Eq. (1) does not predict a transition, as it does not test for the formation of molecules. In Fig. 4, the color coding reveals where molecular behavior is observed (yellow), and where the spectra show polaronic behavior (red to black). It can be seen that the critical interaction strength for the formation of molecules depends only weakly on the impurity concentration $x$.

In conclusion, we have observed Fermi polarons in a novel, attractive Fermi liquid of spin-down impurity atoms immersed in a spin-up Fermi sea. The energy and residue of this quasiparticle was determined and interactions between quasiparticles were found to be weak. Polarons thus emerge as the quasiparticles of a Landau Fermi liquid description of this strongly interacting Fermi mixture. To study first the impurity limit of $N+1$ interacting particles before dealing with the full $N+M$ many-body system will be a fruitful approach for other strongly correlated systems realized with cold atoms. An intriguing question is how the limit of a weakly interacting polaron liquid containing few impurities connects to the physics of a hydrodynamic, balanced Fermi gas containing Cooper pair fluctuations above the critical temperature for superfluidity. In light of our findings, fermion pair condensation could be viewed as condensation of pairs of polarons with opposite spin. This is also suggested by the large normal state interaction measured by quasiparticle spectroscopy on the superfluid state [30].

We would like to thank W. Ketterle, M. Randeria, S. Stringari, B. Svistunov, S. Todadri, and W. Zwerger for helpful discussions, and Aviv Keshet for the computer control system. This work was supported by the NSF, an AFOSR-MURI, and the Alfred P. Sloan Foundation.

[1] L. Landau, Phys. Z. Sowjetunion 3, 644 (1933).

[2] N. Mannella et al., Nature (London) 438, 474 (2005).

[3] P. A. Lee, N. Nagaosa, and X.-G. Wen, Rev. Mod. Phys. 78, 17 (2006).

[4] J. Kondo, Prog. Theor. Phys. 32, 37 (1964).

[5] F. Chevy, Phys. Rev. A 74, 063628 (2006).

[6] C. Lobo et al., Phys. Rev. Lett. 97, 200403 (2006).

[7] C. Schunck et al., Science 316, 867 (2007).

[8] A. Bulgac and M. M. Forbes, Phys. Rev. A 75, 031605(R) (2007).

[9] R. Combescot et al., Phys. Rev. Lett. 98, 180402 (2007).

[10] M. Punk and W. Zwerger, Phys. Rev. Lett. 99, 170404 (2007).

[11] M. Veillette et al., Phys. Rev. A 78, 033614 (2008).

[12] S. Pilati and S. Giorgini, Phys. Rev. Lett. 100, 030401 (2008).

[13] N. V. Prokof'ev and B. V. Svistunov, Phys. Rev. B 77, 020408(R) (2008).

[14] N. V. Prokof'ev and B. V. Svistunov, Phys. Rev. B 77, 125101 (2008).

[15] P. Massignan, G. M. Bruun, and H. T.C. Stoof, Phys. Rev. A 78, 031602(R) (2008).

[16] R. Combescot and S. Giraud, Phys. Rev. Lett. 101, 050404 (2008).

[17] W. Ketterle and M. Zwierlein, in Proceedings of the International School of Physics "Enrico Fermi", Course CLXIV, edited by M. Inguscio, W. Ketterle, and C. Salomon (IOS Press, Amsterdam, 2008), p. 95.

[18] C. H. Schunck et al., Nature (London) 454, 739 (2008).

[19] Y. Shin et al., Phys. Rev. Lett. 99, 090403 (2007).

[20] C. A. Regal et al., Nature (London) 424, 47 (2003).

[21] We do not observe Pauli blocking effects proposed in [15].

[22] See EPAPS Document No. E-PRLTAO-102-040923. For more information on EPAPS, see http://www.aip.org/ pubservs/epaps.html.

[23] W. Schneider, V.B. Shenoy, and M. Randeria, arXiv:0903.3006.

[24] The next order correction due to incoherent scattering, where $a_{\mathrm{fe}}$ is replaced by the full real part of the scattering amplitude, is of order $\left(k_{F} a_{\mathrm{fe}}\right)^{2} \approx 5 \%$ and thus negligible.

[25] Switching roles of states $|1\rangle$ and $|3\rangle$ tests for the influence of final state interactions. They contribute $E_{f}=0.17(1) \epsilon_{F}$ $\left[0.13(1) \epsilon_{F}\right]$ to the peak position when state $|3\rangle(|1\rangle)$ serves as the impurity.

[26] Y. Shin, Phys. Rev. A 77, 041603(R) (2008).

[27] D. E. Sheehy and L. Radzihovsky, Ann. Phys. (N.Y.) 322, 1790 (2007).

[28] Y. Shin et al., Phys. Rev. Lett. 101, 070404 (2008).

[29] S. Sachdev and K. Yang, Phys. Rev. B 73, 174504 (2006).

[30] A. Schirotzek et al., Phys. Rev. Lett. 101, 140403 (2008). 